\title{
LETTERS
}

\section{Clinical COPD phenotypes identified by cluster analysis: validation with mortality}

\section{To the Editors:}

Because forced expiratory volume in $1 \mathrm{~s}$ is a poor descriptor of chronic obstructive pulmonary disease (COPD) heterogeneity [1], interest has emerged regarding the identification of clinically relevant COPD phenotypes [2, 3]. We recently reported the usefulness of applying mathematical models (i.e. principal component and cluster analyses) to multiple variables for the identification of clinical phenotypes in a cohort of COPD subjects [4]. These original analyses allowed the description of four COPD groups, which we called "clinical COPD phenotypes" [4]. This terminology was based on the classical definition of "phenotype", referring to the observable characteristics (including respiratory manifestations and comorbidities) of patients. Recently, a panel of experts proposed the following modified definition for clinical COPD phenotypes: "a single or combination of disease attributes that describes differences between individuals with COPD as they relate to clinically meaningful outcomes (symptoms, exacerbations, response to therapy, rate of disease progression, or death)" $[2,3]$. These authors suggested that, before being referred to as clinical phenotypes, clusters of related patients require longitudinal validation to determine how they differ with respect to important clinical outcomes [2].

Here we report on the longitudinal follow-up of the 322 COPD subjects included in our previous study [4]. The vital status of these subjects was systematically requested on June 19, 2009. Data were obtained for $303(94.1 \%)$ out of the 322 subjects and

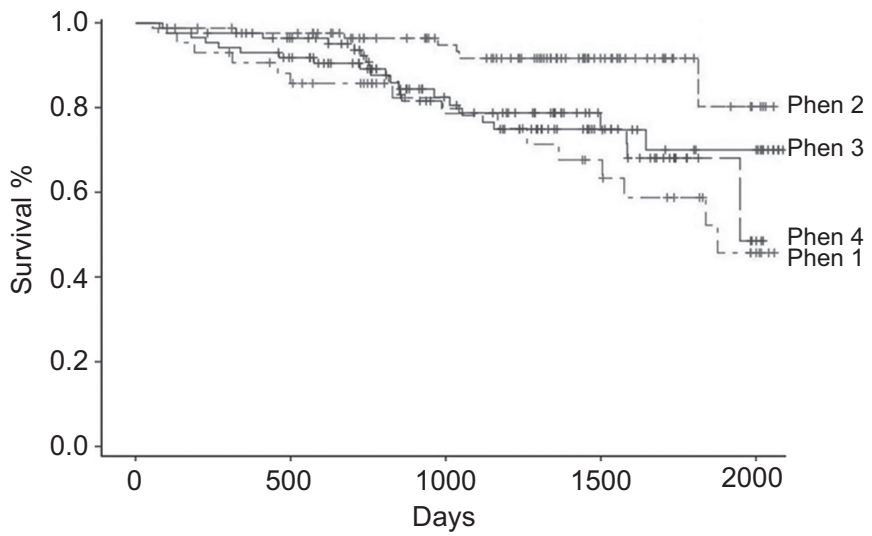

FIGURE 1. Kaplan-Meier analysis of mortality among phenotypes (phen) Subjects in phenotype 2 had the lowest mortality rates (log-rank test with TukeyKramer adjustment for multiple comparisons): phenotype 2 versus phenotype 1 $p=0.003$; phenotype 2 versus phenotype $4, p=0.03$; phenotype 2 versus phenotype $3, p=0.17$. However, no significant difference was observed between the other phenotypes, indicating comparable mortality rates during the (relatively short) follow-up period the median (interquartile range) follow-up duration, which was not significantly different across phenotypes (unpublished data) was 3.35 (2.01-4.25) yrs. During follow-up, 60 (19.8\%) out of 303 subjects died and crude mortality rates were as follows: $15(35 \%)$ out of 43 subjects for phenotype 1 ; seven $(8 \%)$ out of 88 subjects for phenotype $2 ; 17(20 \%)$ out of 87 subjects for phenotype 3 ; and $21(25 \%)$ out of 85 subjects for phenotype 4. Kaplan-Meier analysis of survival by phenotype is presented in figure 1. Subjects in phenotype 2 (older age, mild-to-moderate airflow
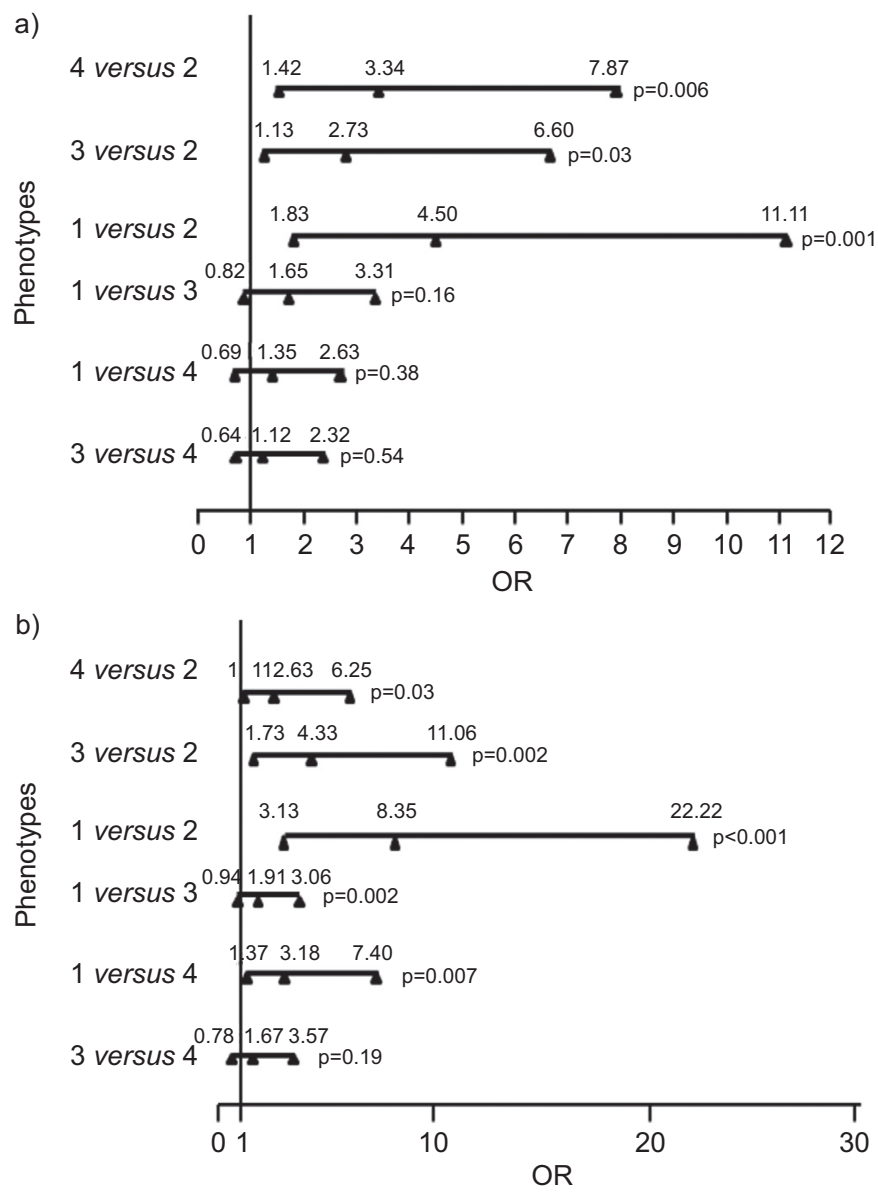

FIGURE 2. Mortality risks in 303 chronic obstructive pulmonary disease subjects with phenotypes and age as covariates. a) Cox model unadjusted for age. b) Cox model adjusted for age. Horizontal bars show odds ratios (OR) and 95\% confidence intervals $(\mathrm{Cls})$ of mortality risks between phenotypes. For example, in the model adjusted for age, subjects in phenotype 1 have an 8.5-fold (95\% Cl 3.1322.22, $\mathrm{p}<0.001$ ) increased risk of mortality when compared with subjects in phenotype 2. 
obstruction and low rates of comorbidities) had a lower mortality, but no significant survival difference was found between phenotypes 1, 3 and 4 . Because subjects belonging to various phenotypes had marked differences in age at inclusion (median age 58-73 yrs) and because the observation period was relatively short, we hypothesised that age of death would be different among the phenotypes. Indeed, median (interquartile range) age of death was: 62 (58-68) yrs for phenotype 1; 77 (66-83) yrs for phenotype 2; 67 (58-69) yrs for phenotype 3; and 76 (74-79) yrs for phenotype 4 . Based on these findings, we analysed mortality risks among phenotypes using Cox models (both unadjusted and adjusted for age) (fig. 2). The unadjusted model confirmed that phenotypes 1, 3 and 4 had a statistically significant two- to four-fold increase in mortality risk when compared with phenotype 2 . In the model adjusted for age, phenotype 1 also had a statistically significant increase in mortality risk compared with phenotype 4 (OR 3.18, 95\% CI 1.37-7.40; $\mathrm{p}=0.007$ ), and a trend towards increased mortality risk compared with phenotype 3 was also observed (OR 1.91, 95\% CI 0.94-3.06; $\mathrm{p}=0.07$ ). However, this trend did not reach statistical significance, probably due to the limited numbers of patients within these phenotypes. No significant difference in mortality risks were observed between phenotype 3 and phenotype 4 .

In conclusion, the longitudinal follow-up identified phenotype 2 as a phenotype with a very low risk of mortality. Additionally, subjects in phenotype 1 (younger subjects with severe airflow limitation, repeated exacerbations, low body mass index and low rates of cardiovascular comorbidities) had the greatest mortality rates and died at a younger age. Thus, these phenotypes actually meet the criteria required to define "clinical phenotypes". Differences in mortality between phenotypes might be even more pronounced over a longer term, but this will have to be confirmed by further survival analyses of the cohort.

Pierre-Régis Burgel*, Nicolas Roche ${ }^{\#}$, Jean-Louis Paillasseur", Isabelle Tillie-Leblond ${ }^{+}$, Pascal Chanez ${ }^{\S}$, Roger Escamilla ${ }^{f}$, Isabelle Court-Fortune ${ }^{* *}$, Thierry Perez ${ }^{+}$, Philippe Carré ${ }^{* \uparrow}$ and Denis Caillaud $^{\text {"थ }}$ on behalf of the INITIATIVES BPCO scientific committee
*Service de Pneumologie, Hôpital Cochin, AP-HP and Paris Descartes University, "Service de Pneumologie, Hôpital de l'Hôtel Dieu, AP-HP and Paris Descartes University, Paris, 'Clindatafirst, St Quentin en Yvelines, ${ }^{+}$Service de Pneumologie, Hôpital Calmette, Lille, ${ }^{\S}$ Dépt des Maladies Respiratoires, AP-HM, Université de la Méditerranée, Marseille, ${ }^{f}$ Clinique des voies respiratoires Hopital Larrey, Toulouse, **Service de Pneumologie, CHU Saint Etienne, Saint Etienne, ${ }^{\# \#}$ Service de Pneumologie, Hôpital Antoine Gayraud, Carcassonne, and "Service de Pneumologie, Hôpital Gabriel Montpied, CHU Clermont-Ferrand, France.

Correspondence: P-R. Burgel, Service de Pneumologie, Hôpital Cochin, Assistance Publique Hôpitaux de Paris, 27 rue du Faubourg St Jacques, 75679 Paris Cedex 14, France. E-mail: pierre-regis.burgel@cch.aphp.fr

Support Statement: This work was funded by unrestricted grants from Boehringer Ingelheim and Pfizer (both Paris, France).

Statement of Interest: A statement of interest for this study can be found at www.erj.ersjournals.com/site/misc/statements. xhtml

\section{REFERENCES}

1 Agusti A, Calverley PM, Celli B, et al. Characterisation of COPD heterogeneity in the ECLIPSE cohort. Respir Res 2010; 11: 122.

2 Han MK, Agusti A, Calverley PM, et al. Chronic obstructive pulmonary disease phenotypes: the future of COPD. Am J Respir Crit Care Med 2010; 182: 598-604.

3 Agusti A, Celli B. Avoiding confusion in COPD: from risk factors to phenotypes to measures of disease characterisation. Eur Respir J 2011; 38: 749-751.

4 Burgel PR, Paillasseur JL, Caillaud D, et al. Clinical COPD phenotypes: a novel approach using principal component and cluster analyses. Eur Respir J 2010; 36: 531-539.

\section{A new point of care cotinine test for saliva to identify and monitor smoking habit}

\section{To the Editors:}

We welcome the paper by CAPONNETTO et al. [1], in which they evaluated a nicotine-free inhalator as part of a smoking cessation programme. In the randomised controlled trial, they found no significant improvement with the inhalator, but further analysis of the data showed a significant increase in cessation rate among those with strong and very strong behavioural dependence as measured by questionnaire. While this was a good attempt to find inexpensive means to improve smoking cessation, we question their methodology to assess smoking dependency and abstinence.
Questionnaires such as those used for the Fagerström Dependence Score and the Glover-Nilsson Smoking Behavioural Questionnaire are subject to bias and intentional and unintentional underreporting. Also, smoke topography, the way a smoker uses a cigarette, depth of inhalation and nicotine metabolism are extremely variable. The best way of assessing smoking behaviour and nicotine intake is by biochemical analysis. This paper did utilise expired-air carbon monoxide (CO) monitoring, but this has significant drawbacks; it is not specific to tobacco intake (being influenced by atmospheric $\mathrm{CO}$ ) and it has a short half-life of $3 \mathrm{~h}$, so assessing smoking behaviour over a 6 - to 8 -h period. Nicotine metabolites, such as cotinine, are judged to be the best biochemical 